\title{
Symanzik improvement of lattice QCD with four flavors of Wilson quarks
}

\author{
ALPHA Collaboration \\ Fatih Tekin $^{\mathrm{a}, *}$, Rainer Sommer ${ }^{\mathrm{b}}$, Ulli Wolff ${ }^{\mathrm{a}}$ \\ a Institut für Physik, Humboldt Universität, Newtonstr. 15, 12489 Berlin, Germany \\ b NIC, DESY, Platanenallee 6, 15738 Zeuthen, Germany
}

\section{A R T I C L E I N F O}

\section{Article history:}

Received 20 November 2009

Accepted 24 November 2009

Available online 27 November 2009

Editor: A. Ringwald

\begin{abstract}
A B S T R A C T
We have determined the non-perturbative $\mathcal{O}(a)$-improvement coefficient $c_{\mathrm{sw}}$ for four flavors of Wilson quarks with the plaquette gauge action in a range of $\beta \geqslant 5.0$. The data are fitted with several Padé approximation formulae to get an impression of the stability. A small extrapolation below $\beta=5.0$ seems acceptable.
\end{abstract}

(C) 2009 Elsevier B.V. All rights reserved.

\section{Introduction}

The lattice regularization of QCD is a powerful tool to nonperturbatively study QCD in the low energy region. The numerical implementation involves however a finite lattice spacing $a$ which has to be removed in the continuum limit. The rate of approaching the continuum limit will depend on the details of the lattice formulation. A systematic way to reduce the discretization effects order by order in $a$ is the Symanzik improvement programme [1-3] for on-shell quantities [4,5]. In the case of Wilson fermions, Sheikholeslami and Wohlert [6] have shown that for reducing the lattice artefacts from $\mathcal{O}(a)$ to $\mathcal{O}\left(a^{2}\right)$ only one additional dimension five operator in the Lagrangian is needed. To achieve this acceleration of the continuum limit non-perturbatively the coefficient $c_{\mathrm{SW}}$ of the corresponding operator has to be determined in numerical simulations. In the quenched case [7], the ALPHA Collaboration has found [8-10] that the non-perturbative result for $c_{\mathrm{sw}}$ deviates significantly from the one-loop perturbative value $[6,11]$. Furthermore, the effect of two species of dynamical fermions on $c_{\mathrm{sw}}$ was also studied by the ALPHA Collaboration and the difference to the quenched case was clearly visible [12]. The effect of a third flavor was studied by the CP-PACS and JLQCD Collaborations [13] with the result that $c_{\mathrm{sw}}\left(g_{0}^{2}\right)$ is not very much affected by it and their result is very close to the two flavor values of the ALPHA Collaboration which can be seen in Fig. 4. Our aim in this Letter is to calculate $c_{\mathrm{sw}}$ non-perturbatively for four flavors in the Schrödinger functional scheme.

\footnotetext{
* Corresponding author.

E-mail addresses: fatih@physik.hu-berlin.de (F. Tekin), rainer.sommer@desy.de (R. Sommer), uwolff@physik.hu-berlin.de (U. Wolff).
}

The Letter is organized as follows. First we want to give a brief reminder of $\mathcal{O}(a)$ improved lattice QCD and briefly discuss the improvement condition for $c_{\mathrm{sw}}$. After discussing some features of the algorithmic implementation we will give a summary about the simulation parameters and the raw data. The procedure how to determine $c_{\mathrm{sw}}$ from the raw data will be described next. Finally we will give the conclusions.

\section{Improvement condition} action

Our starting point for $\mathcal{O}(a)$ improved lattice $\mathrm{QCD}$ is the fermion

$S_{\mathrm{f}}=a^{4} \sum_{x} \bar{\psi}(x)\left[D+m_{0}\right] \psi(x)$,

where $a$ is the lattice spacing and $m_{0}$ is the bare quark mass. The matrix $D$ is the Wilson-Dirac operator [14]

$D=\frac{1}{2}\left[\left(\nabla_{\mu}^{*}+\nabla_{\mu}\right) \gamma_{\mu}-a \nabla_{\mu}^{*} \nabla_{\mu}\right]$

with the lattice covariant forward and backward derivatives $\nabla_{\mu}$ and $\nabla_{\mu}^{*}$. The leading order lattice artefacts in on-shell quantities which are calculated with this action are linear in $a$. However, the leading order discretization effects may be canceled by adding the so-called Sheikholeslami-Wohlert term to the action [6]

$D_{\text {improved }}=D+c_{\mathrm{sw}} \frac{i a}{4} \sigma_{\mu \nu} \mathcal{F}_{\mu \nu}$.

The lattice field strength tensor $\mathcal{F}_{\mu \nu}$ is defined as in [15] and $\sigma_{\mu \nu}=\frac{i}{2}\left[\gamma_{\mu}, \gamma_{\nu}\right]$. The coefficient $c_{\mathrm{sw}}$ in Eq. (3) is a function of the bare coupling $g_{0}$ and if it is chosen properly, $D_{\text {improved }}$ becomes 
the on-shell $\mathcal{O}(a)$ improved lattice Wilson-Dirac operator. For a complete cancellation of the $\mathcal{O}(a)$ effects ${ }^{1}$ in correlation functions, the local composite fields that enter also have to be improved [15]. In our case, such composite fields are the isovector axial current $A_{\mu}^{a}(x)$ and the pseudo-scalar density $P^{a}(x)$. It turns out that only the isovector axial current needs an improvement because there is no dimension four operator with the same behavior as $P^{a}$ under the symmetries of the lattice theory. The $\mathcal{O}(a)$ improvement requires the combination

$\left(A_{\mathrm{I}}\right)_{\mu}^{a}=A_{\mu}^{a}+a \cdot c_{\mathrm{A}} \frac{1}{2}\left(\partial_{\mu}^{*}+\partial_{\mu}\right) P^{a}$

where $A_{\mu}^{a}$ and $P^{a}$ are given by

$A_{\mu}^{a}(x)=\bar{\psi}(x) \gamma_{\mu} \gamma_{5} \frac{\tau^{a}}{2} \psi(x)$,

$P^{a}(x)=\bar{\psi}(x) \gamma_{5} \frac{\tau^{a}}{2} \psi(x)$,

$\partial_{\mu}, \partial_{\mu}^{*}$ are the forward and backward difference operators and $\tau^{a}$ are Pauli matrices acting on one pair among the four degenerate flavors. The improvement coefficient $c_{\mathrm{A}}$ is known in perturbation theory [16] and from non-perturbative determinations for $N_{\mathrm{f}}=0$ [7] and $N_{\mathrm{f}}=2[17,18]$. As explained in [7,12], we introduce the unrenormalized PCAC quark mass

$m\left(x_{0}\right)=\frac{\frac{1}{2}\left(\partial_{0}+\partial_{0}\right) f_{\mathrm{A}}\left(x_{0}\right)+c_{\mathrm{A}} a \partial_{0}^{*} \partial_{0} f_{\mathrm{P}}\left(x_{0}\right)}{2 f_{\mathrm{P}}\left(x_{0}\right)}$

where the correlation functions $f_{\mathrm{A}}$ and $f_{\mathrm{P}}$ contain $A_{\mu}^{a}$ and $P^{a}$ and are given by (2.1) and (2.2) of [7]. A second mass $m^{\prime}$ can be defined in the same way as Eq. (7) but with the primed correlation functions $f_{\mathrm{A}}^{\prime}$ and $f_{\mathrm{P}}^{\prime}((2.5)$ and (2.6) of [7]). The unprimed and primed correlation functions are related to each other by a time reflection in the Schrödinger functional. Since the boundary conditions

$\left.U(x, k)\right|_{x_{0}=0}=\exp \left\{a C_{k}\right\} ; \quad C_{k}=\frac{i}{6 L} \operatorname{diag}(-\pi, 0, \pi)$,

$\left.U(x, k)\right|_{x_{0}=T}=\exp \left\{a C_{k}^{\prime}\right\} ; \quad C_{k}^{\prime}=\frac{i}{6 L} \operatorname{diag}(-5 \pi, 2 \pi, 3 \pi)$

are such that $C_{k}$ and $C_{k}^{\prime}$ are not the same, $f_{\mathrm{X}}$ and $f_{\mathrm{X}}^{\prime}$ also differ. Since with PCAC we have, however, inserted an 'operator identity', all $m\left(x_{0}\right), m^{\prime}\left(y_{0}\right)$ differ only at the level of $\mathcal{O}\left(a^{2}\right)$ effects in the improved theory. We could hence for some choice, such as $x_{0}=$ $y_{0}=\frac{T}{2}$, impose $m-m^{\prime}=0$ as one condition for the proper choice of $c_{\mathrm{SW}}$ and $c_{\mathrm{A}}$. Because the coefficient $c_{\mathrm{A}}$ is a priori not known it is advantageous however to first eliminate this parameter and define a quark mass $M$ independent of $c_{\mathrm{A}}$ which agrees with the quark mass $m$ up to $\mathcal{O}\left(a^{2}\right)$ effects. For this purpose we name the partial contributions

$r\left(x_{0}\right)=\frac{\left(\partial_{0}^{*}+\partial_{0}\right) f_{\mathrm{A}}\left(x_{0}\right)}{4 f_{\mathrm{P}}\left(x_{0}\right)}$,

$s\left(x_{0}\right)=\frac{a \partial_{0}^{*} \partial_{0} f_{\mathrm{P}}\left(x_{0}\right)}{2 f_{\mathrm{P}}\left(x_{0}\right)}$

and rewrite the mass $m$ as

$m\left(x_{0}\right)=r\left(x_{0}\right)+c_{A} s\left(x_{0}\right)$.

With an analogous definition for $m^{\prime}$, a quark mass $M$ can than be written in the following way

1 This refers to the massless theory which we consider in connection with the Schrödinger functional renormalization scheme.

$$
\begin{aligned}
M\left(x_{0}, y_{0}\right) & =m\left(x_{0}\right)-s\left(x_{0}\right) \frac{m\left(y_{0}\right)-m^{\prime}\left(y_{0}\right)}{s\left(y_{0}\right)-s^{\prime}\left(y_{0}\right)} \\
& =r\left(x_{0}\right)-s\left(x_{0}\right) \frac{r\left(y_{0}\right)-r^{\prime}\left(y_{0}\right)}{s\left(y_{0}\right)-s^{\prime}\left(y_{0}\right)}
\end{aligned}
$$

In this combination, which in the improved theory differs from $m$ by $\mathcal{O}\left(a^{2}\right)$ only, $c_{\mathrm{A}}$ drops out. Now, we define $M^{\prime}\left(x_{0}, y_{0}\right)$ analogously and could require that the difference

$\Delta M\left(\frac{3}{4} T, \frac{1}{4} T\right)=M\left(\frac{3}{4} T, \frac{1}{4} T\right)-M^{\prime}\left(\frac{3}{4} T, \frac{1}{4} T\right)$

has to vanishes for our value of $c_{\mathrm{sw}}$. The choice $\left(x_{0}, y_{0}\right)=\left(\frac{3}{4} T, \frac{1}{4} T\right)$ is one possible choice [7] for the argument of $\Delta M$. For the quark mass $M$ itself we choose $\left(x_{0}, y_{0}\right)=\left(\frac{1}{2} T, \frac{1}{4} T\right)$ [7]. In order to reproduce the tree level value of $c_{\mathrm{sw}}$ exactly for finite $a$, we finally impose the improvement condition

$\Delta M=\Delta M^{(0)}$

where $\Delta M^{(0)}$ is the tree level value of perturbation theory in the $\mathcal{O}(a)$ improved theory. For $L / a=8$ one finds for example [7]

$\left.a \Delta M^{(0)}\right|_{M=0, c_{\mathrm{sw}}=1}=0.000277$

As discussed in [19], improvement coefficients possess a unique perturbative expansion but non-perturbatively they are themselves ambiguous by cutoff terms and thus depend on the choice of the improvement conditions. Of course, for QCD these uncertainties amount to cutoff effects beyond the order that is improved, $\mathcal{O}\left(a^{2}\right)$ in the case at hand. In principle one then has to determine improvement coefficients for one fixed set of conditions as functions of $g_{0}$ at constant physics, i.e. fixing all scale ratios except $a$ that shrinks with $g_{0}$. For the Schrödinger functional this would in particular require constant $L / r_{0}$ as $g_{0}$ is lowered. For practical reasons we fix however $L / a$ instead and refer the reader to Section I.2.4.1 of [19] for a detailed discussion. The replacement of zero by the small tree-level value on the right-hand side of Eq. (16) guarantees that our definition has the correct limit for $g_{0} \rightarrow 0$.

\section{Simulations}

Our simulations for $N_{\mathrm{f}}=4$ are based on an adaptation of TAO codes - suitable for APE computers [20] - used earlier by the ALPHA Collaboration for $N_{\mathrm{f}}=2$ studies. An ordinary HMC algorithm [21] has been implemented with symmetric even-odd preconditioning [22-24] and the Sexton-Weingarten integration scheme [25]. Mass preconditioning [26,27] was not enforced as we expect that the gain for the Schrödinger functional with the parameters envisaged here would not be so significant [28]. For the sake of convenience, from here on, we will set the lattice spacing $a$ to one.

\subsection{Algorithm}

The fermionic determinant for an even number of flavors can be represented by pseudo-fermion fields $\phi_{i}, \phi_{i}^{\dagger}, i=1, \ldots, N_{\mathrm{f}} / 2$. The partition function then reads for $N_{\mathrm{f}}=4$

$Z=\int D U D \phi_{1}^{\dagger} D \phi_{1} D \phi_{2}^{\dagger} D \phi_{2} \mathrm{e}^{-S_{\mathrm{G}}-\sum_{i=1,2} \phi_{i}^{\dagger}\left(Q Q^{\dagger}\right)^{-1} \phi_{i}}$

where $S_{\mathrm{G}}$ is Wilson's plaquette gauge action [14] and $Q$ is related to the Dirac matrix $M$ by 
$Q=\gamma_{5} M=\gamma_{5}\left(\begin{array}{ll}M_{\mathrm{ee}} & M_{\mathrm{eo}} \\ M_{\mathrm{oe}} & M_{\mathrm{oo}}\end{array}\right)$.

The key idea of the even-odd preconditioning $[22,23]$ is to divide the lattice sites into even and odd sites according to the sum over the coordinates $\sum_{\mu=0}^{3} x_{\mu}$. If it is even for the lattice site $x$, the site is called even otherwise it is called odd. Following this strategy, the Dirac matrix $M$ decomposes into the block form in Eq. (18). The components are given as follows [29]

$$
\begin{aligned}
M_{x, x^{\prime}}= & \left(1+T_{x, x}\right) \delta_{x, x^{\prime}}-\kappa \sum_{\mu}\left[\left(1-\gamma_{\mu}\right) U_{\mu}(x) \delta_{x+\hat{\mu}, x^{\prime}}\right. \\
& \left.+\left(1+\gamma_{\mu}\right) U_{\mu}^{\dagger}(x-\hat{\mu}) \delta_{x-\hat{\mu}, x^{\prime}}\right] .
\end{aligned}
$$

The matrices $T_{x, x}$

$T_{x, x}=\frac{i}{2} c_{\mathrm{sw}} \kappa \sigma_{\mu \nu} \mathcal{F}_{\mu \nu}(x)$

vanish if the improvement coefficient $c_{\mathrm{sw}}$ is set to zero and the submatrices $M_{\mathrm{ee}}$ and $M_{\mathrm{oo}}$ then become equal to the unit matrix. In our case however, we consider the $\mathcal{O}(a)$ improved Sheikholeslami-Wohlert action where the coefficient $c_{\mathrm{sw}}$ is determined non-perturbatively for $N_{\mathrm{f}}=4$. Furthermore, the submatrices of $M$ possess the following properties

$\begin{array}{ll}M_{\mathrm{ee}}^{\dagger}=M_{\mathrm{ee}}, & M_{\mathrm{oo}}^{\dagger}=M_{\mathrm{oo}}, \\ M_{\mathrm{eo}}^{\dagger}=\gamma_{5} M_{\mathrm{oe}} \gamma_{5}, & M_{\mathrm{oe}}^{\dagger}=\gamma_{5} M_{\mathrm{eo}} \gamma_{5} .\end{array}$

The origin of the algorithmic acceleration by even-odd preconditioning lies in the factorization of the determinant. In this context, two possibilities appear. We may either factorize out only $M_{\mathrm{ee}}$ or $M_{\mathrm{oo}}$ (asymmetric), or second, we extract both factors (symmetric). The derivation of both versions is very similar. The key point is the calculation of a determinant of matrices like Eq. (18) using its Schur complement. For a general matrix which consist of submatrices, the determinant can be calculated in the following way

$\operatorname{det}\left(\begin{array}{ll}A & B \\ C & D\end{array}\right)=\operatorname{det}\left\{A D-A C A^{-1} B\right\}$.

In our case, the determinant of $Q=\gamma_{5} M$ leads to

$\operatorname{det} Q=\operatorname{det}\left\{M_{\mathrm{ee}}\right\} \operatorname{det}\left\{M_{\mathrm{oo}}\right\} \operatorname{det}\{\hat{Q}\}$

for the symmetric even-odd preconditioning, where

$\hat{\mathrm{Q}}=\gamma_{5}\left(1-M_{\mathrm{oo}}^{-1} M_{\mathrm{oe}} M_{\mathrm{ee}}^{-1} M_{\mathrm{eo}}\right)$.

In our numerical implementation, we only use the above described symmetric even-odd preconditioning but there is no fundamental problem to implement the asymmetric even-odd preconditioned version. However, it should be kept in mind that the authors of [30] found that the performance of HMC with symmetric evenodd preconditioning is roughly $30 \%$ better than the HMC algorithm with asymmetric even-odd preconditioning. Due to the decomposition in Eq. (24), only the odd components $\phi_{0}$ of the fields $\phi$ appear. With symmetric preconditioning the partition function for four flavors now reads

$Z=\int D U D \phi_{01}^{\dagger} D \phi_{01} D \phi_{02}^{\dagger} D \phi_{02} \mathrm{e}^{-S_{\mathrm{G}}[U]-S_{\mathrm{det}}[U]-S_{\mathrm{pf}}\left[U, \phi_{\mathrm{oi}}^{\dagger}, \phi_{\mathrm{oi}}\right]}$

with the gauge part $S_{\mathrm{G}}[U]$ and

$$
\begin{aligned}
& S_{\mathrm{det}}=N_{\mathrm{f}}\left[\ln \operatorname{det}\left\{M_{\mathrm{ee}}\right\}+\ln \operatorname{det}\left\{M_{\mathrm{oo}}\right\}\right], \\
& S_{\mathrm{pf}}=\sum_{i=1,2} \phi_{\mathrm{o} i}^{\dagger}\left(\hat{\mathrm{Q}} \hat{\mathrm{Q}}^{\dagger}\right)^{-1} \phi_{\mathrm{o} i} .
\end{aligned}
$$

\subsection{Simulation parameters and raw results}

The simulations were performed in the Schrödinger functional scheme [8-10] with periodic boundary conditions for the spatial extension and Dirichlet boundary conditions in the temporal direction. This means the phase $\theta$ was here set to zero in all runs. The data were obtained on hypercubic Euclidean $16 \times 8^{3}$ lattices. The $\mathcal{O}$ (a) improvement of the Schrödinger functional requires additional improvement terms at the boundaries. However, since PCAC is an operator relation, these terms are irrelevant for a correct determination of $c_{\mathrm{sw}}$. Nevertheless, we have chosen them as follows. The pure gauge part of the action acquires a weight $w(p)=c_{\mathrm{t}}\left(g_{0}\right)$ of time-like plaquettes $p$ attached to the boundary planes for which we have inserted the 2-loop value [31]

$$
\begin{aligned}
c_{\mathrm{t}}\left(g_{0}\right)= & 1+\left[-0.08900+0.0191410 N_{\mathrm{f}}\right] g_{0}^{2} \\
& +\left[-0.0294+0.002 N_{\mathrm{f}}+0 N_{\mathrm{f}}^{2}\right] g_{0}^{4},
\end{aligned}
$$

and for the fermionic improvement coefficient $\tilde{c}_{\mathrm{t}}\left(g_{0}\right)$ [16] we take the 1-loop perturbative value [32]

$\tilde{c}_{\mathrm{t}}\left(g_{0}\right)=1-0.01795 g_{0}^{2}$.

In all our simulations the trajectory length was kept fixed to one. We chose the intervals in $\beta$ similar to [12]. The mean acceptance rate was around $90 \%$ and we performed 4400 trajectories per value of $\beta$ and $c_{\mathrm{sw}}$ on average. A large part of our computations ran on APEmille machines with 128 processors each. For some values of $\beta$ and $c_{\mathrm{sw}}$ we also used apeNEXT crates with 256 processors. The summary table of the measurements can be found in Appendix A.

\section{Determination of $c_{s w}$}

The numerical procedure for the determination of $c_{\mathrm{sw}}$ involves the following main steps:

1. Compute $\Delta M$ and $M$ for several $\kappa$ at fixed $g_{0}^{2}$ (or $\beta$ ) and $c_{\text {sw. }}$. Then interpolate linearly in $M$ to find $\Delta M$ at vanishing quark mass $M=0$.

2. For fixed $g_{0}^{2}$, repeat step 1 for several values of $c_{\mathrm{sw}}$ and find $c_{\mathrm{SW}}^{*}$ which solves Eq. (15) by a linear fit in $c_{\mathrm{sw}}$.

3. Repeat the preceding steps for a sufficient range of $g_{0}^{2}$ and fit these data with an appropriate function to represent the smooth functional dependence of $c_{\mathrm{sw}}$ on $g_{0}^{2}$.

This procedure is computer time demanding because for each value of $\beta$ and for each value of $c_{\mathrm{sw}}$ of Table 2, we would need several runs, at least three, for interpolating $\Delta M$ in $M$ to $M=0$. To save computer time, we modified this method slightly. As discussed in the determination of $c_{\mathrm{sw}}$ for $N_{\mathrm{f}}=0,2$ [7,12], the weak dependence of $\Delta M$ on $M$ holds also at $N_{\mathrm{f}}=4$. For one set of parameters $\beta$ and $c_{\mathrm{sw}}$, we checked the dependence explicitly (Fig. 1). Since $\Delta M$ depends weakly on $M$, we contented ourselves with determining $\Delta M$ for some $|M|<0.03$ [12] and used these values of $\Delta M$ as an approximation for $\Delta M$ at $M=0$. A typical result is shown in Fig. 2. We performed simulations from $\beta=12$ to $\beta=5.0$. For each $\beta$, we calculated the observables $\Delta M$ and $M$ at least for three different values of $c_{\mathrm{Sw}}$ in such a way that the condition $|M|<0.03$ held and that $\Delta M$ had a change of sign. The linear interpolation $\Delta M=s\left(c_{\mathrm{sw}}-c_{\mathrm{sW}}^{*}\right)+\Delta M^{(0)}$ yields the desired values $c_{\mathrm{sw}}^{*}$ shown in Table 1 . We also tried to go below $\beta=5.0$ but at $\beta=4.8$, we were not able to locate a significant sign change of $\Delta M$ in our data and therefore after some attempts, we decided to stop searching.

The CP-PACS and JLQCD Collaborations computed $c_{\mathrm{sw}}$ for $N_{\mathrm{f}}=3$ in the Schrödinger functional setup of lattice QCD with the plaque- 


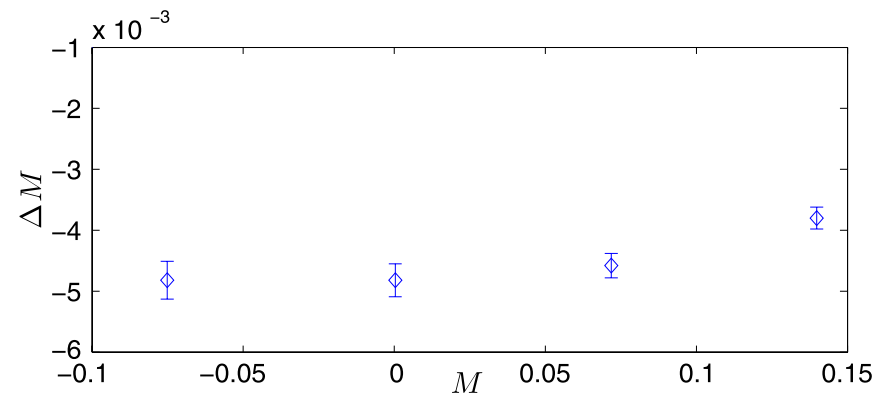

Fig. 1. Mass dependence of $\Delta M$ at $\beta=5.0$ and $c_{\mathrm{sw}}=2.4$. The errors of $M$ are smaller than the symbol sizes.

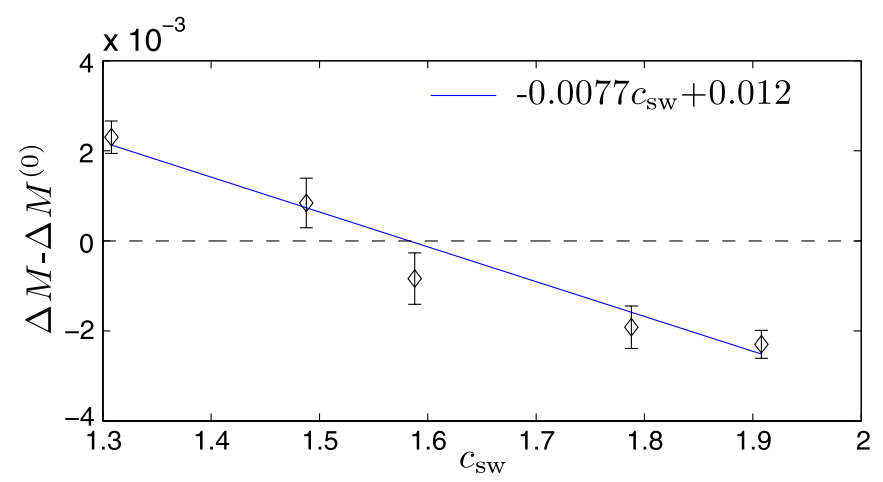

Fig. 2. Determination of $c_{\mathrm{sw}}$ at $\beta=5.4$. The desired value $c_{\mathrm{sw}}^{*}$ is located at the point where the fit curve (solid) passes through zero (dashed).

Table 1

Results of the linear interpolation.

\begin{tabular}{clll}
\hline$\beta$ & $c_{\mathrm{sw}}^{*}$ & $\beta$ & $c_{\mathrm{sw}}^{*}$ \\
\hline 12 & $1.1429(39)$ & 6.0 & $1.463(19)$ \\
9.6 & $1.1895(62)$ & 5.7 & $1.554(17)$ \\
7.4 & $1.2955(76)$ & 5.4 & $1.583(25)$ \\
6.8 & $1.3375(94)$ & 5.2 & $1.614(28)$ \\
6.3 & $1.389(12)$ & 5.0 & $1.717(31)$ \\
\hline
\end{tabular}

tte gauge action [13]. They found that the result for three flavors is very close to the two flavor result [12]. In addition they calculated $c_{\mathrm{sw}}$ with four flavors for $\beta=9.6$ and found $c_{\mathrm{sW}}^{*}=1.1954(48)$ in good agreement with our $c_{\mathrm{SW}}^{*}=1.1895(62)$.

After obtaining the proper values $c_{\mathrm{sw}}^{*}$ which satisfy the improvement condition (15), we want to represent and interpolate our data by a simple Padé formula, appropriate for the achieved precision, which also incorporates the known 1-loop perturbative result. The solution that we want to advocate here for $N_{\mathrm{f}}=4$ is

$c_{\mathrm{sw}}\left(g_{0}^{2}\right)=\frac{1-0.1372 g_{0}^{2}-0.1641 g_{0}^{4}+0.1679 g_{0}^{6}}{1-0.4031 g_{0}^{2}}$,

$$
0 \leqslant g_{0}^{2} \leqslant 1.2
$$

This curve appears as Fit1 in Fig. 3. The two other lines Fit2 and Fit3 include one and two more powers in the numerator. The deviation of the different fit formulae in the range $g_{0}^{2} \in[0,1.2]$ $(\beta=[12.0,5.0])$ is negligible and beyond $g_{0}^{2}=1.2$, Fit 1 and Fit2 are almost the same down to $\beta=4.5$ but Fit 3 deviates slightly. Our non-perturbatively determined formula (30) for $c_{\mathrm{sw}}$ with four flavors is valid down to $\beta=5.0\left(g_{0}^{2}=1.2\right)$ but may perhaps be used to $\beta=4.5$ within a small uncertainty.

To conclude in Fig. 4, we juxtapose our new data and fit formula at $N_{\mathrm{f}}=4$ to those known for $N_{\mathrm{f}}=0,2,3$.

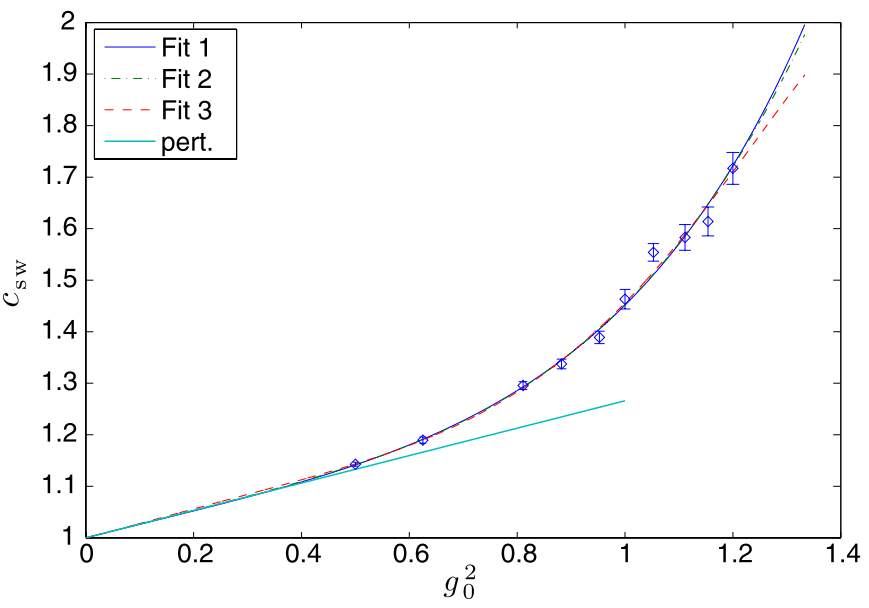

Fig. 3. Comparison of different Padé-approximation formulae for our data.

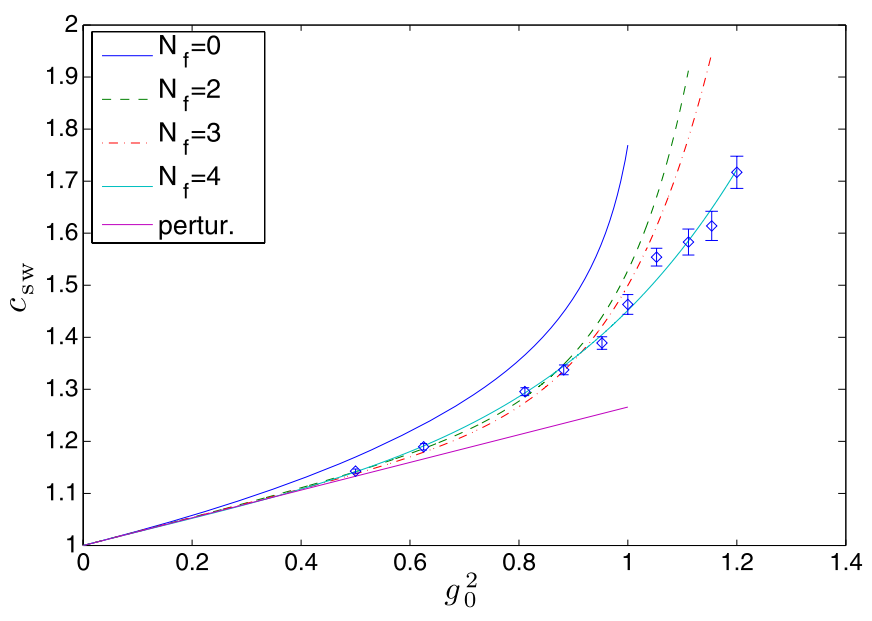

Fig. 4. Summary plot of all known $c_{\mathrm{sw}}\left(g_{0}^{2}, N_{\mathrm{f}}\right)$ for the plaquette gauge action.

\section{Conclusions}

In the present Letter, we have performed simulations for the calculation of the improvement coefficient $c_{\mathrm{sw}}$ for four flavors of Wilson fermions in a range of $\beta \geqslant 5.0$ and give Eq. (30) as a suitable parameterization of our data. We compared three simpleminded different Padé-approximation formulae for our data and could show that a small extrapolation of $c_{\mathrm{sw}}$ beyond $\beta=5.0$ to $\beta=4.5$ with the main formula (30) may still be acceptable. An immediate use of $c_{\mathrm{sw}}$ will be the determination of the nonperturbative running of the Schrödinger functional coupling for $N_{\mathrm{f}}=4$ massless flavors, extending the present knowledge which comprises $N_{\mathrm{f}}=0$ [7], $N_{\mathrm{f}}=2$ [12] and $N_{\mathrm{f}}=3$ [13].

\section{Acknowledgements}

This work is part of the ALPHA Collaboration research program. We thank NIC for allocating computer time on the APE computers at DESY, Zeuthen and the staff of the computer center at Zeuthen for their support. Fatih Tekin thanks Oliver Witzel for numerous fruitful discussions about the details of the ALPHA code and for useful suggestions. This work is supported by the Deutsche Forschungsgemeinschaft (DFG) in the framework of SFB/TR 09 and by the European Community through EU Contract No. MRTN-CT2006-035482, "FLAVIAnet". 


\section{Appendix A}

Table of data:

Table 2

Summary table of the measurements.

\begin{tabular}{|c|c|c|c|c|}
\hline$\beta$ & $\kappa$ & $c_{\mathrm{sw}}$ & $M$ & $\Delta M$ \\
\hline \multirow[t]{3}{*}{12} & 0.130280 & 1.028654 & $0.000224(75)$ & $0.001735(89)$ \\
\hline & 0.129897 & 1.128654 & $-0.001510(74)$ & $0.000468(84)$ \\
\hline & 0.129449 & 1.228654 & $-0.001371(82)$ & $-0.000827(86)$ \\
\hline \multirow[t]{3}{*}{9.6} & 0.131516 & 1.140488 & $-0.00004(11)$ & $0.00077(12)$ \\
\hline & 0.131164 & 1.170488 & $0.00595(11)$ & $0.00053(11)$ \\
\hline & 0.131164 & 1.250488 & $-0.00853(11)$ & $-0.00040(12)$ \\
\hline \multirow[t]{4}{*}{7.4} & 0.134626 & 1.163222 & $-0.00196(20)$ & $0.00169(19)$ \\
\hline & 0.133753 & 1.263222 & $0.00072(20)$ & $0.00069(15)$ \\
\hline & 0.132989 & 1.363222 & $0.00018(20)$ & $-0.00036(16)$ \\
\hline & 0.132349 & 1.443222 & $0.00027(20)$ & $-0.00162(17)$ \\
\hline \multirow[t]{5}{*}{6.8} & 0.135638 & 1.209613 & $0.00128(30)$ & $0.00169(26)$ \\
\hline & 0.135082 & 1.299613 & $-0.00642(32)$ & $0.00033(26)$ \\
\hline & 0.134896 & 1.309613 & $-0.00333(29)$ & $0.00079(24)$ \\
\hline & 0.133813 & 1.409613 & $0.00320(29)$ & $-0.00000(24)$ \\
\hline & 0.133056 & 1.509613 & $-0.00141(30)$ & $-0.00204(22)$ \\
\hline \multirow[t]{4}{*}{6.3} & 0.137098 & 1.239058 & $-0.00129(38)$ & $0.00199(24)$ \\
\hline & 0.136018 & 1.339058 & $0.00142(36)$ & $0.00077(27)$ \\
\hline & 0.135028 & 1.439058 & $0.00140(36)$ & $-0.00075(27)$ \\
\hline & 0.134028 & 1.550580 & $-0.00251(31)$ & $-0.00115(24)$ \\
\hline \multirow[t]{3}{*}{6.0} & 0.138358 & 1.250000 & $-0.00090(85)$ & $0.00270(30)$ \\
\hline & 0.136669 & 1.387912 & $0.00214(47)$ & $0.00022(48)$ \\
\hline & 0.134375 & 1.587912 & $0.00677(43)$ & $-0.00123(34)$ \\
\hline \multirow[t]{5}{*}{5.7} & 0.140327 & 1.250000 & $-0.0082(16)$ & $0.00369(38)$ \\
\hline & 0.138229 & 1.387912 & $0.00034(63)$ & $0.00107(43)$ \\
\hline & 0.137008 & 1.487912 & $0.00081(55)$ & $0.00076(48)$ \\
\hline & 0.135685 & 1.587912 & $0.00564(63)$ & $-0.00032(37)$ \\
\hline & 0.133940 & 1.754350 & $-0.00004(38)$ & $-0.00156(28)$ \\
\hline \multirow[t]{5}{*}{5.4} & 0.141417 & 1.307912 & $-0.00032(77)$ & $0.00258(36)$ \\
\hline & 0.139111 & 1.487912 & $-0.00712(89)$ & $0.00112(55)$ \\
\hline & 0.137815 & 1.587912 & $-0.00762(70)$ & $-0.00056(57)$ \\
\hline & 0.135028 & 1.787912 & $-0.00117(69)$ & $-0.00164(47)$ \\
\hline & 0.133775 & 1.907912 & $-0.00854(39)$ & $-0.00202(31)$ \\
\hline \multirow[t]{6}{*}{5.2} & 0.143363 & 1.307912 & $-0.0004(14)$ & $0.00196(48)$ \\
\hline & 0.140628 & 1.487912 & $-0.00032(87)$ & $0.00076(42)$ \\
\hline & 0.139206 & 1.587912 & $-0.00326(69)$ & $0.00102(39)$ \\
\hline & 0.138147 & 1.655891 & $0.00162(94)$ & $0.00028(47)$ \\
\hline & 0.136248 & 1.787912 & $0.00030(91)$ & $-0.00084(65)$ \\
\hline & 0.134556 & 1.907912 & $0.00372(62)$ & $-0.00189(38)$ \\
\hline \multirow[t]{6}{*}{5.0} & 0.146056 & 1.307912 & $0.0051(25)$ & $0.00252(62)$ \\
\hline & 0.142554 & 1.507912 & $0.0021(13)$ & $0.00125(41)$ \\
\hline & 0.138141 & 1.787912 & $-0.0053(11)$ & $0.00102(68)$ \\
\hline & 0.136527 & 1.885463 & $0.0009(11)$ & $0.00004(55)$ \\
\hline & 0.135039 & 2.000000 & $-0.00826(90)$ & $-0.00070(59)$ \\
\hline & 0.129603 & 2.400000 & $0.00033(42)$ & $-0.00482(27)$ \\
\hline \multirow[t]{7}{*}{4.8} & 0.145928 & 1.500000 & $0.0083(54)$ & $-0.0002(12)$ \\
\hline & 0.142201 & 1.700000 & $-0.0022(31)$ & $-0.0000(11)$ \\
\hline & 0.138295 & 1.910000 & $0.0017(13)$ & $0.00060(62)$ \\
\hline & 0.137971 & 1.930000 & $0.0031(12)$ & $-0.00054(48)$ \\
\hline & 0.136844 & 2.000000 & $0.0008(11)$ & $-0.00044(50)$ \\
\hline & 0.135327 & 2.100000 & $-0.00492(87)$ & $-0.00116(44)$ \\
\hline & 0.132358 & 2.300000 & $-0.00550(72)$ & $-0.00244(41)$ \\
\hline
\end{tabular}

\section{References}

[1] K. Symanzik, Some topics in quantum field theory, Presented at 6th Int. Conf. on Mathematical Physics, Berlin, West Germany, 11-21 August 1981.

[2] K. Symanzik, Nucl. Phys. B 226 (1983) 187.

[3] K. Symanzik, Nucl. Phys. B 226 (1983) 205.

[4] M. Lüscher, P. Weisz, Phys. Lett. B 158 (1985) 250

[5] M. Lüscher, P. Weisz, Commun. Math. Phys. 97 (1985) 59.

[6] B. Sheikholeslami, R. Wohlert, Nucl. Phys. B 259 (1985) 572.

[7] Martin Lüscher, Stefan Sint, Sommer Rainer, Peter Weisz, Ulli Wolff, Nucl. Phys. B 491 (1997) 323.

[8] Martin Lüscher, Rajamani Narayanan, Peter Weisz, Ulli Wolff, Nucl. Phys. B 384 (1992) 168.

[9] Stefan Sint, Nucl. Phys. B 421 (1994) 135.

[10] Stefan Sint, Nucl. Phys. B 451 (1995) 416.

[11] R. Wohlert, Improved continuum limit lattice action for quarks, DESY 87/069, 1987.

[12] Karl Jansen, Rainer Sommer, Nucl. Phys. B 530 (1998) 185.

[13] N. Yamada, et al., Phys. Rev. D 71 (2005) 054505.

[14] Kenneth G. Wilson, Phys. Rev. D 10 (8) (October 1974) 2445.

[15] Martin Lüscher, Stefan Sint, Rainer Sommer, Peter Weisz, Nucl. Phys. B 478 (1996) 365.

[16] M. Lüscher, P. Weisz, Nucl. Phys. B 479 (1996) 429.

[17] Michele Della Morte, Roland Hoffmann, Rainer Sommer, JHEP 0503 (2005) 029.

[18] Michele Della Morte, Rainer Sommer, Shinji Takeda, Phys. Lett. B 672 (2009) 407.

[19] Rainer Sommer, Non-perturbative QCD: Renormalization, $\mathrm{O}(a)$-improvement and matching to heavy quark effective theory, hep-lat/0611020, 2006.

[20] R. Alfieri, et al., Nucl. Phys. B (Proc. Suppl.) 94 (2001) 846.

[21] Simon Duane, A.D. Kennedy, Brian J. Pendleton, Duncan Roweth, Phys. Lett. B 195 (2) (1987) 216.

[22] Rajan Gupta, Apoorva Patel, Clive F. Baillie, Gerald Guralnik, Gregory W. Kilcup, Stephen R. Sharpe, Phys. Rev. D 40 (6) (September 1989) 2072.

[23] Thomas A. Degrand, Pietro Rossi, Comput. Phys. Commun. (ISSN 00104655) 60 (2) (1990) 211.

[24] O. Witzel, Non-Hermitian Polynomial Hybrid Monte Carlo, PhD thesis, Humboldt University Berlin, 2008.

[25] J.C. Sexton, D.H. Weingarten, Nucl. Phys. B (ISSN 0550-3213) 380 (3) (1992) 665.

[26] Martin Hasenbusch, Phys. Lett. B 519 (2001) 177.

[27] M. Hasenbusch, K. Jansen, Nucl. Phys. B 659 (2003) 299.

[28] Michele Della Morte, et al., Comput. Phys. Commun. 156 (2003) 62.

[29] Karl Jansen, Chuan Liu, Comput. Phys. Commun. 99 (1997) 221.

[30] S. Aoki, R. Burkhalter, M. Fukugita, S. Hashimoto, K.-I. Ishikawa, N. Ishizuka, Y. Iwasaki, K. Kanaya, T. Kaneko, Y. Kuramashi, M. Okawa, T. Onogi, S. Tominaga, N. Tsutsui, A. Ukawa, N. Yamada, T. Yoshié, Phys. Rev. D 65 (9) (April 2002) 094507.

[31] Achim Bode, Peter Weisz, Ulli Wolff, Nucl. Phys. B 576 (2000) 517.

[32] Stefan Sint, Peter Weisz, Nucl. Phys. B 502 (1997) 251. 